\title{
Cortical GABAergic Interneurons Transiently Assume a Sea Urchin-Like Nonpolarized Shape before Axon Initiation
}

\author{
Emi Yamasaki, ${ }^{1}$ Daisuke H. Tanaka, ${ }^{1}$ Yuchio Yanagawa, ${ }^{2}$ and Fujio Murakami ${ }^{1}$ \\ ${ }^{1}$ Graduate School of Frontier Biosciences, Osaka University, Suita, Osaka 560-8531, Japan, and 2Department of Genetics and Behavioural Neuroscience, \\ Gunma University Graduate School of Medicine, Maebashi 371-8511, Japan
}

\begin{abstract}
Mature neurons polarize by extending an axon and dendrites. In vitro studies of dissociated neurons have demonstrated that axons are initiated from a nonpolarized stage. Dissociated hippocampal neurons form four to five minor neurites shortly after plating but then one of them starts to elongate rapidly to become the future axon, whereas the rest constitutes the dendrites at later stages. However, neuroepithelial cells as well as migrating neurons in vivo are already polarized, raising the possibility that mature neurons inherit the polarities of immature neurons of neuroepithelial or migrating neurons. Here we show that the axon of interneurons in mouse cortical explant emerges from a morphologically nonpolarized shape. The morphological maturation of cortical interneurons labeled by electroporation at an embryonic stage was analyzed by time-lapse imaging during the perinatal stage. In contrast to earlier stages, most interneurons at this stage show sea urchin-like nonpolarized shapes with alternately extending and retracting short processes. Abruptly, one of these processes extends to give rise to an outstandingly long axon-like process. Given that the interneurons exhibit typical polarized shapes during embryonic development, the present results suggest that axon-dendrite polarity develops from a nonpolarized intermediate stage.
\end{abstract}

\section{Introduction}

Neurons typically show polarized morphology, defined as a single axon and several dendrites. A fundamental question is how is this axon-dendrite polarity established. Although there is evidence that an extrinsic directional cue can induce axons (Adler et al., 2006), developing neurons can acquire polarity in culture in the absence of any extrinsic signal directional gradients (for review, see Arimura and Kaibuchi, 2007). This issue has been extensively studied using hippocampal cells in culture: these cells, shortly after plating, extend lamellipodia, which then develop into several short, immature neurites (Dotti et al., 1988; Goslin and Banker, 1989). After a period of alternating elongation and retraction, one process acquires an axonal nature. Once a single process acquires the axonal nature, none of the remaining processes become an axon. These findings indicate that axon-dendrite polarity determination is an intrinsically regulated selection in which the "winner" among equivalent early processes becomes an axon.

However, most postmitotic neurons in situ are already polarized at the time of generation, because neuroepithelial cells have basoapical polarity. Thus, neurons that arise from neuroepithe-

Received March 25, 2010; revised Aug. 25, 2010; accepted Sept. 1, 2010.

This work was supported by Grant-in-Aid for Scientific Research 17100005 from the Ministry of Education, Culture, Sports, Science, and Technology, Japan. We thank T. Kobayashi for pCAGGS-GAP-EGFP, Dr. Y. Hatanaka for pCAG-EGFP and pCAG-dsRed2, Drs. Y. Tanabe, K. Yamauchi, and S. C. Fujita for critically reading this manuscript, and Dr. Takaki Miyata for useful discussion.

Correspondence should be addressed to Fujio Murakami, Graduate School of Frontier Biosciences, Osaka University, Yamadaoka 1-3, Suita, 0saka 565-0871, Japan. E-mail: murakami@fbs.osaka-u.ac.jp.

D. H. Tanaka's present address: Department of Anatomy, Keio University School of Medicine, 35 Shinanomachi, Shinjuku-ku, Tokyo 160-8582, Japan.

DOI:10.1523/JNEUROSCI.1527-10.2010

Copyright $\odot 2010$ the authors $\quad 0270-6474 / 10 / 3015221-07 \$ 15.00 / 0$ lial cells could in some way inherit basoapical polarity, which could then lead to the establishment of axon-dendritic polarity. This idea is supported by observations of early development of neurons in fixed preparations (Stensaas, 1967a,b; Hinds and Hinds, 1974, 1978; Wentworth and Hinds, 1978; Martínez et al., 1992; Wilcock et al., 2007) in which basal processes appear to turn into axons. Moreover, neurons or neuronal precursors that have just been separated from neuroepithelial cells by cell division are also polarized with morphologies of migrating cells (Lambert de Rouvroit and Goffinet, 2001; Tsai and Gleeson, 2005), indicating that neurons are in some way already polarized in vivo before extending axons.

Nevertheless, the idea that axon-dendrite polarity emerges from a nonpolarized stage in vivo cannot be ruled out, because there is insufficient literature on the sequential changes in morphology during the transformation of migrating neurons into axon-dendrite polarized mature ones in the developing brain. To address this issue, we focused on cortical interneurons because their generation occurs at a location different from that of maturation, which enables us to analyze the process of maturation separately from early neurogenesis. These neurons are generated in ganglionic eminences in the basal forebrain (Anderson et al., 1997; Tamamaki et al., 1997) and migrate to the neocortex. Previously, we found that interneurons labeled by electroporation at embryonic day 12.5 (E12.5) execute multidirectional tangential migration in the marginal zone (MZ) of the neocortex for a protracted period of time (Tanaka et al., 2003, 2006, 2009). During this period, many interneurons change their direction of migration by changing their polarity of migration (Tanaka et al., 2009). Thereafter, these neurons descend to the cortical plate (CP) (Tanaka et al., 2009). 

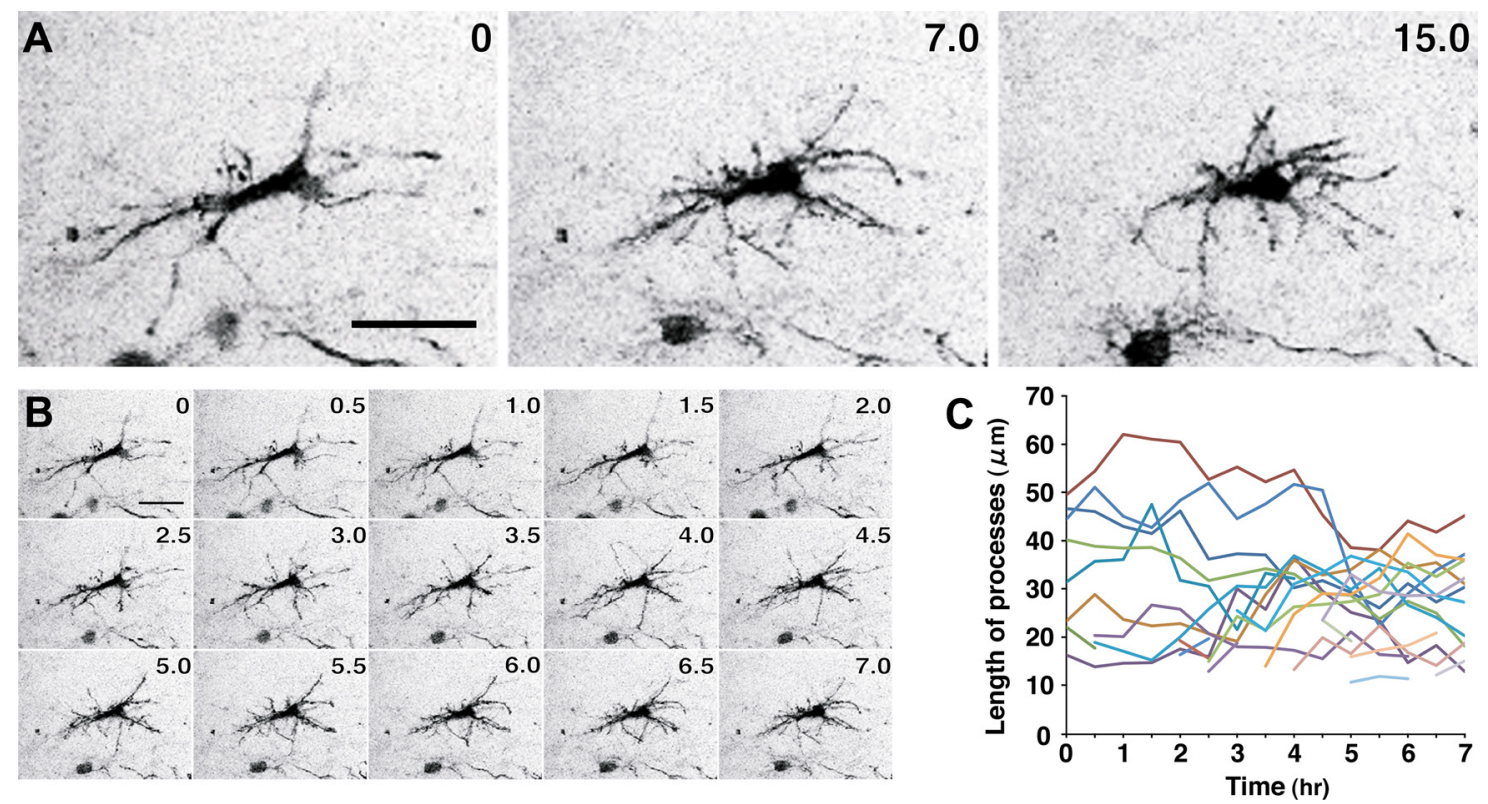

Figure 1. Sea urchin-like cells alternately extend and retract short processes. $A, B$, Time sequence of confocal images of a sea urchin-like cell. Imaging began at $P 0.5$. Elapsed time after start of observation (hours) is indicated on the top right corner of each photograph. $\boldsymbol{C}$, Length of processes of cell shown in $\boldsymbol{B}$ plotted against elapsed time. Different colors represent different processes. Extensions and retractions can be seen. Scale bar, $30 \mu \mathrm{m}$.

Here we performed long-time ( $>40 \mathrm{~h}$ ) live imaging of cortical interneurons at the stage when they reach the CP by using organ culture preparations that recapitulate their in vivo development. We found that cortical interneurons transiently assume a nonpolarized shape during early postnatal development alternately extending and retracting many short processes. One of these processes rapidly elongates far beyond the length of the alternating extensions and retractions, suggesting that polarity begins from nonpolarized stage.

\section{Materials and Methods}

Animals. ICR mice of both sexes were used in most of the experiments. GAD67-GFP $(\Delta$ neo) mice (Tamamaki et al., 2003) were used in some experiments in which interneurons were labeled by Discosoma red 2 (DsRed2). They were also used to confirm that the laminated structure of the cortex is retained in our culture condition. Noon on the day of vaginal plug detection was defined E0.5; E19.5 was defined as postnatal day $0(\mathrm{P} 0)$. All experiments were done in accordance with institutional guidelines.

Electroporation. In or ex utero electroporation into the medial ganglionic eminence was performed on E12.5 embryos. Plasmid vectors used were $p C A G G S-D s R e d 2, p C A G-E G F P$, and $p C A G G S-G A P-E G F P$. Unless otherwise stated, data shown in figures and movies were from cells labeled with $p C A G G S-G A P-E G F P$, but essentially the same results were obtained when using $p C A G G S-D s R e d 2$. The methods for electroporation were similar to those described previously (Tanaka et al., 2006), except that 30-34 V electric pulses were delivered at $800 \mathrm{~ms}$ intervals for ex utero electroporation and $60 \mathrm{~V}$ pulses at $600 \mathrm{~ms}$ intervals for in utero electroporation.

Time-lapse imaging. The head of E18.5 or P0.5 electroporated mice was removed and placed in a plastic dish filled with HBSS with the dorsal surface of the brain exposed. Then the brain was chilled by placing the dish in a freezer $\left(-20^{\circ}\right)$ for $5-10$ min to slightly harden the brain. Brains were then cut into two halves at an intermediate level along the rostrocaudal axis in HBSS (Nissui) on a vibrating-blade microtome (VT-1000; Leica Microsystems). The rostral half of the cut brain was mounted onto membrane inserts (Millicell-CM PICMORG50; Millipore) coated with collagen gels with the sectioned side down. The foot of the mounted tissue was covered with collagen gels and maintained in Opti-MEM I reduced-serum medium containing $2.5 \%$ heat-inactivated fetal bovine serum, $2.5 \%$ heat-inactivated horse serum, $1 \% \mathrm{~N} 2$ supplement (all from Invitrogen), $20 \mathrm{~mm}$ D-glucose, and $20 \mu \mathrm{g} / \mathrm{ml}$ streptomycin (Sigma). After culturing the hemisected brain for $4-6 \mathrm{~h}$, the preparation was transferred to a glass-bottom dish (FluoroDish FD35-100; World Precision Instruments) in a temperature- and gas-controlled incubation chamber $\left(5 \% \mathrm{CO}_{2}, 95 \% \mathrm{O}_{2}\right.$ at $37^{\circ} \mathrm{C}$ ) fitted onto a confocal microscope (FV1000; Olympus) that was equipped with a motor-driven $x-y$ stage (FC-101G; Sigma Koki).

Labeled cells were viewed through a $20 \times$ objective (numerical aperture 0.45 ). Images were collected with the confocal microscope using a $488 \mathrm{~nm}$ excitation wavelength and 505-525 nm emission filter for green fluorescent protein (GFP) or a $543 \mathrm{~nm}$ excitation wavelength and $560 \mathrm{~nm}$ emission filter for DsRed. Images were captured from multiple points of the cerebral cortex by moving a motor-driven $x-y$ stage regulated by the aid of multiarea time-lapse software (FV10-ASW version 1.6b; Olympus). Time-lapse imaging was performed for $36-43 \mathrm{~h}$ at $30 \mathrm{~min}$ time intervals. At each time point, a stack of images was created from a series of four to five consecutive images taken along the $z$-axis at $10 \mu \mathrm{m}$ intervals. The tissue boundary was identified by referring to differential interference contrast (DIC) images. The laminated structure of the cortex was retained during the course of imaging. Brightness and contrast were adjusted for every frame using Adobe Photoshop 7.0 or CS4 software. Movies were assembled using Adobe Photoshop CS4 or ImageReady 7.0 software.

Staining and quantitative analysis of fixed samples. E18.5 to P2.5 brains were dissected from electroporated mice and immersion fixed in $4 \%$ paraformaldehyde (PFA) in phosphate buffer $(\mathrm{PB})$ overnight at $4^{\circ} \mathrm{C}$, cut coronally at $50 \mu \mathrm{m}$ on a vibrating-blade microtome, and stained with an anti-GFP antibody. P7-P21 mice were transcardially perfused with 4\% PFA in PB under deep anesthesia with sodium pentobarbitone (Nembutal; $100-200 \mathrm{mg} / \mathrm{kg}$ body weight; Abbott Laboratories) before immersion fixation. Sections were sampled from the intermediate one-third of the neocortex along the rostrocaudal axis, extending caudally to the level of the caudal end of the hippocampus. The slices were transferred to 48 -well plates and incubated in biotin-conjugated anti-GFP (1:500; Rockland Immunochemicals) in $0.3 \%$ Triton X-100/PBS for $2-6 \mathrm{~d}$ at $4^{\circ} \mathrm{C}$, followed by $5 \mathrm{~h}$ incubation in Alexa Fluor 488 streptavidin (1:200; Invitrogen) at room temperature (RT). Then sections were counterstained with TOPRO-3 iodide (1:2000; Invitrogen) at RT. The slices were then divided into three groups: rostral, intermediate, and caudal. A set of three con- 

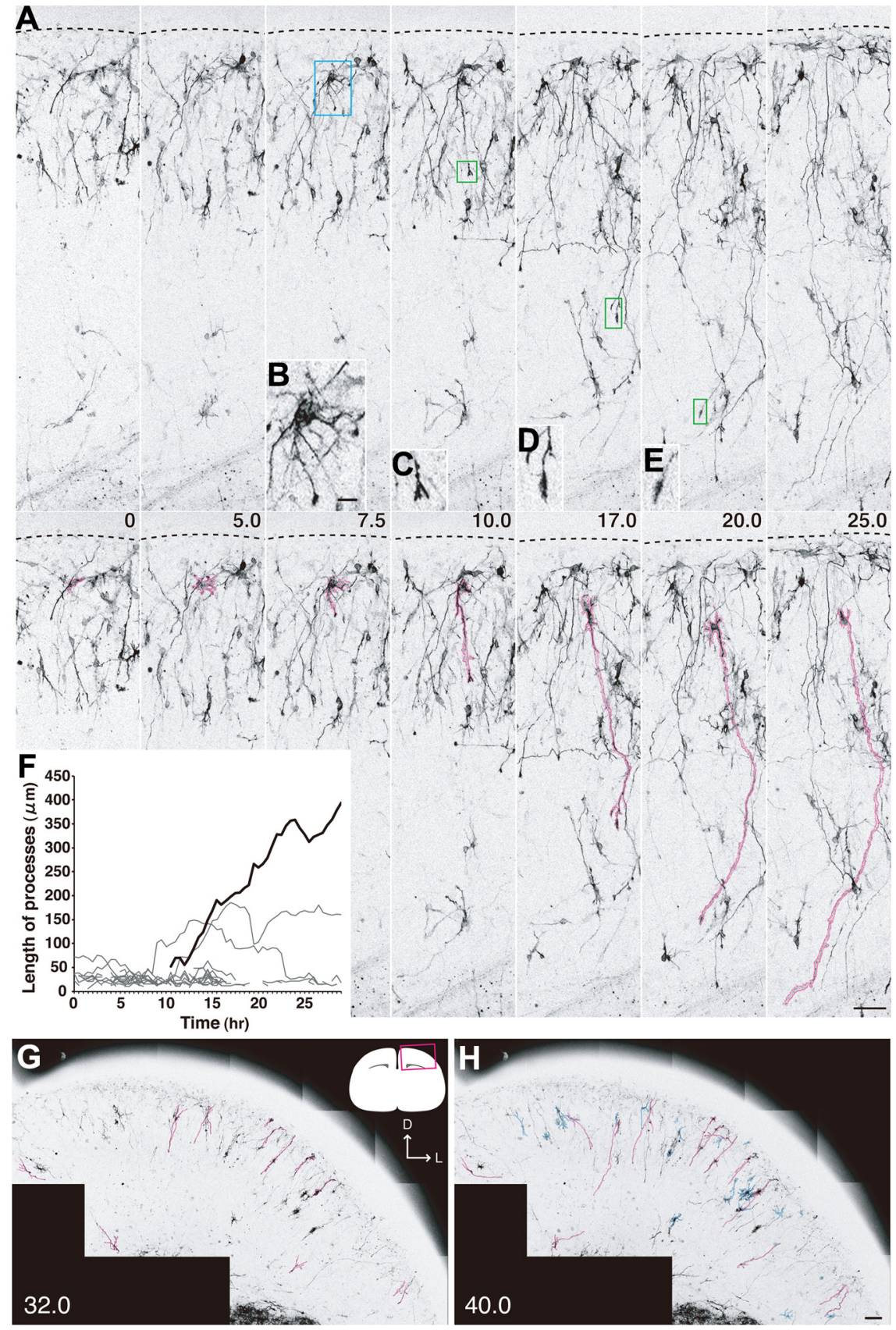

Figure 2. Extension of an axon-like process from a sea urchin-like cell. $\boldsymbol{A}$, Time sequence of confocal images of a cell that initiated an axon-like process during time-lapse imaging. Time-lapse imaging began at P0.5. Panels in top and bottom rows show the same cell, but in the bottom row, an axon-like process is marked in pink. Elapsed time after the onset of imaging (hours) is indicated on the top right corner of the bottom panels. Dashed lines indicate the pial surface. $\boldsymbol{B}-\boldsymbol{E}$, Growing tips of axon-like processes. High magnification of the areas demarcated by rectangles in $\boldsymbol{A}$. $\boldsymbol{B}$ corresponds to the blue rectangle in $\boldsymbol{A}$ and shows a sea urchin like-cell that has just initiated an axon-like process headed by a growth cone. $\boldsymbol{C}-\boldsymbol{E}$, Growing tips of the axon-like process demarcated by green rectangles in $\boldsymbol{A}$. $\boldsymbol{F}$, Time course of the extension of several processes from a sea urchin-like cell. Many short processes repeatedly extend and retract, but one continues to extend almost monotonically (thick black line). Note that two other processes extend up to $150-200 \mu \mathrm{m}$ but fail to extend farther. This cell corresponds to the one shown in supplemental Movie 4 (available at www.jneurosci.org as supplemental material). $\mathbf{G}, \boldsymbol{H}$, Lowmagnification images of the lateral cortex showing the morphologies of interneurons extending axon-like processes (magenta). $G$, Image taken $32 \mathrm{~h}$ after the onset of imaging; $\boldsymbol{H}$, image taken $40 \mathrm{~h}$ after the onset of imaging. Comparison of these two images demonstrates extension of long processes. Scale bars: $\boldsymbol{A}, 30 \mu \mathrm{m} ; \boldsymbol{B}-\boldsymbol{E}, 10 \mu \mathrm{m} ; \boldsymbol{G}, \boldsymbol{H}, 100 \mu \mathrm{m}$. D, Dorsal; L, lateral.

secutive sections was arbitrarily chosen from each of the group and was subjected to confocal microscopy (FV1000; Olympus). Confocal images taken from the three consecutive sections were superimposed by adjusting the tissue border. In some experiments, a motor-driven light microscope stage was used to capture images at multiple points in a wide field but with a high magnification. The images from each point were assembled thereafter. The following filter sets were used with the indicated wave lengths for excitation laser line and emission filters (in nm): GFP(green), 488/ (505-525); TO-PRO-3(far red), 633/(650) with FV1000. To identify the tissue border, DIC images were captured simultaneously with the fluorescent images. Adobe Photoshop 7.0 software was used for images processing described above.

For quantitative analysis, the second section in the triplet was picked up. Labeled cells were counted between the line drawn tangentially to the medial surface of the lateral ventricle and the one drawn perpendicularly to the lateral surface of the cortex from the edge of the corticostriatal sulcus.

To assess the orientation of GABAergic neurons, a line was drawn from the center of the cell body to the tip of the longest process emanating from the cell body. Then, the length of this line and the angle between this line and a line drawn perpendicular to the pial surface at the point where the former intersects with the pial surface were measured. To define the center of the cell body, two lines were drawn tangentially to the cell body surface, each on its opposite side and parallel with each other, and the midpoint between the point of two tangency was defined as its center. When the angles $(x)$ were $0^{\circ}<x<45^{\circ}, 135^{\circ}<x<225^{\circ}$, $315^{\circ}<x<360^{\circ}$, the direction was defined as radial. When the angles $(x)$ were $45^{\circ}<x<$ $135^{\circ}$ and $225^{\circ}<x<315^{\circ}$, the direction was defined as tangential. The layer distribution of labeled cells between the ventricular zone and the marginal zone of cerebral cortex was also examined by nuclear staining.

For immunostaining of axons with axonal markers, $50-\mu \mathrm{m}$-thick sections were cut from a P2.5 mouse brain on a vibrating-blade microtome and reacted with a mixture of mouse anti-SMI-312 monoclonal antibody (1:1000; Covance) and goat biotin-conjugated antiGFP (1:500; Rockland Immunochemicals) for at least $2 \mathrm{~d}$ at $4^{\circ} \mathrm{C}$, washed with PBS, followed by a mixture of cyanine 3 (Cy3)-conjugated anti-mouse IgG (1:200; Jackson ImmunoResearch) and Alexa Fluor 488-streptavidin (1: 200; Invitrogen) for $4-6 \mathrm{~h}$ at RT.

To determine transmitter phenotypes, P21 fixed brains were cryoprotected by immersion in $30 \%$ sucrose and cut coronally at $20 \mu \mathrm{m}$. The sections were mounted on slides and washed with PBS. The sections were then incubated in PBS with $0.5 \%$ Triton X-100 and 5-10\% normal goat serum (NGS) for $2-5 \mathrm{~h}$ at RT. This was followed by incubation in the primary antibody diluted in PBS with $0.5 \%$ Triton X-100 and $5 \%$ NGS for $2 \mathrm{~d}$ at RT. The primary antibodies used were as follows: rabbit antiparvalbumin (PV) (1:2000 to 1:4000; Swant), rat anti-somatostatin (SST) (1:100; Millipore Bioscience Research Reagents, and rabbit anticalretinin (CR) (1:2000; Swant). The sections were incubated for $3 \mathrm{~h}$ at RT in the secondary antibody diluted in $1 \%$ NGS in PBS with $0.5 \%$ Triton X-100. The secondary antibodies used were either goat Cy3conjugated anti-rabbit IgG (1:100) or goat Cy3-conjugated anti-rat IgG 
(1:200) (both from Jackson ImmunoResearch). For nuclear staining, all sections were incubated in $0.03 \%$ 4,6-diamidino-2-phenylindole (Nacalai Tesque) in PBS for $30 \mathrm{~min}$ at RT. Images were captured using a CCD camera (AxioCam; Carl Zeiss) attached to an epifluorescence microscope (BX60; Olympus).

\section{Results}

Sea urchin-like cells

Many cortical interneurons labeled by electroporation at E12.5 are observed in the MZ at E18.5 but become predominantly localized at the $\mathrm{CP}$ from $\mathrm{P} 2$ onward (Tanaka et al., 2009). This suggests that these interneurons begin to terminate migration and mature during E18.5 to P2. Therefore, we began time-lapse imaging at E18.5 or P0.5 and thereafter examined the dynamics of labeled cells in the CP.

A major population of labeled cells in the CP exhibited a multipolar morphology (Fig. 1 $A$ ) and alternately extended and retracted numerous short processes (Fig. 1A-C) (supplemental Movie 1, available at www.jneurosci.org as supplemental material), although rapidly migrating cells extending a leading process were also observed. Such multipolar cells did not substantially change the position of their cell body (Fig. 1A,B) (supplemental Movie 1, available at www.jneurosci. org as supplemental material), unlike those with a simple bipolar morphology observed at earlier stages (Tanaka et al., 2003, 2006, 2009). This suggests that these cells are nearing the completion of their migration, in agreement with a previous report that found cortical interneurons lose motility during the early postnatal stage (Bortone and Polleux, 2009). Because of their peculiar features, we termed such cells sea urchin-like cells.

\section{Elongation of an axon-like process}

During the course of real-time imaging, we noted that, unlike most processes, which repeatedly extended and retracted, one process from the sea urchin-like cells continued to elongate during the period of observation or until it exited the observation field. Figure $2 \mathrm{~A}$ exemplifies such an extension. This neuron showed multiple processes extending and retracting for $\sim 7.5 \mathrm{~h}$ from the onset of observation [Fig. $2 A(t=0$ to $t=7.5), B]$. However, suddenly one began to elongate almost monotonically up to the end of the observation period (Fig. 2A) (supplemental Movie 2, available at www.jneurosci.org as supplemental material). The thin and long morphology of this process together with its distinct behavior from others strongly suggests that this is a prospective axon. Indeed, growth-cone-like

A

C
E18.5

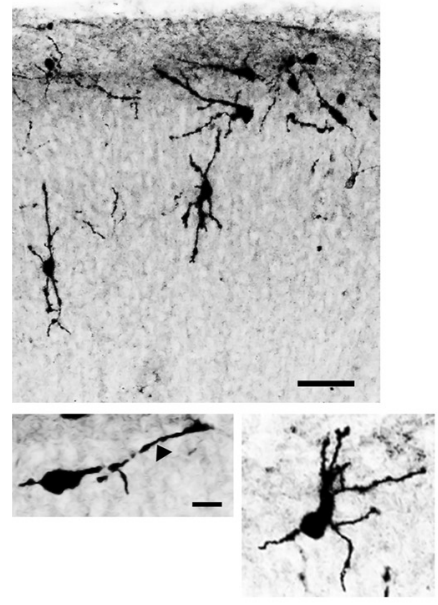

P1.5 D

P2.5
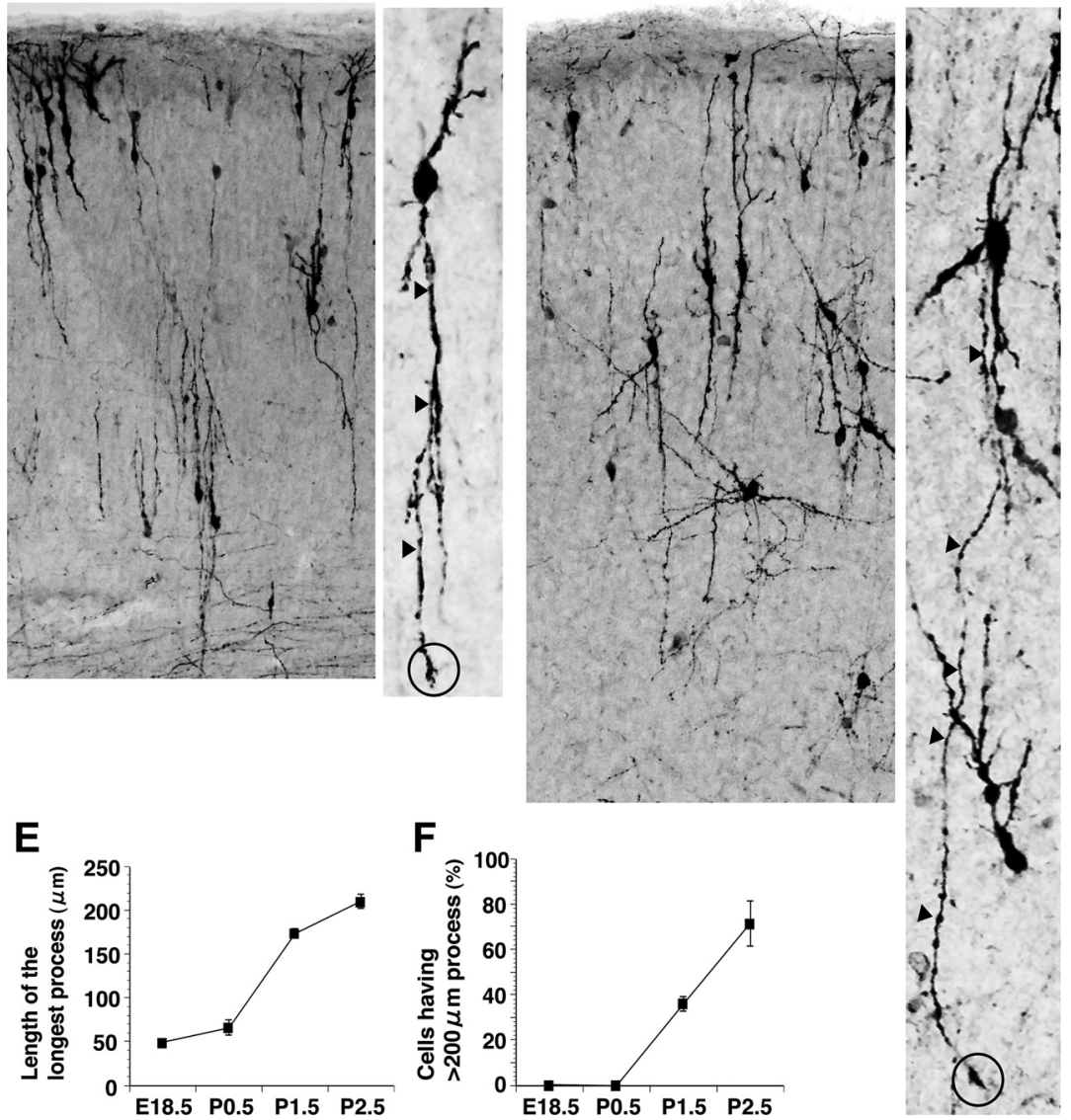

Figure 3. GABAergic interneurons observed in perinatal mouse neocortex in vivo. Neurons in the CP and marginal zone are shown $\boldsymbol{A}-\boldsymbol{D}$, Confocal images of samples fixed in coronal sections at E18.5 (A), P0.5 (B), P1.5 (C), and P2.5 (D). Most labeled cells in the marginal zone show migrating neurons with a bipolar shape $(\boldsymbol{A}$, bottom left), but those in the (P primarily showed a multipolar morphology atE18.5 ( $\boldsymbol{A}$, bottom right). At P0.5, although labeled cells extend longer processes, many show multipolar morphology. At later stages, cellular morphologies become more complex (D). In $\boldsymbol{A}-\boldsymbol{D}$, the largest panel in each shows a low-magnification view of the representative samples. The bottom two panels in $\boldsymbol{A}$ and right panels in $\boldsymbol{B}-\boldsymbol{D}$ show high-power pictures. $\boldsymbol{E}$, Length of the longest process becomes larger as development proceeds. $\boldsymbol{F}$, Proportion of labeled cells bearing a long $(>200 \mu \mathrm{m})$ process markedly increases during postnatal development. In $\boldsymbol{E}$ and $\boldsymbol{F}$, numbers of neurons analyzed were 833 at E18.5, 745 at P0.5, 915 at P1.5, and 702 at P2.5. Scale bars: top panel in $\boldsymbol{A}$, left panels in $\boldsymbol{B}-\boldsymbol{D}, 50 \mu \mathrm{m}$; bottom panels in $\boldsymbol{A}$, right panels in $\boldsymbol{B}-\boldsymbol{D}, 10 \mu \mathrm{m}$.

swellings were observed at the leading edge of such elongating processes (Fig. 2C-E). Such an extension of processes from labeled cells occurred in a widespread manner across the developing neocortex (Fig. 2G,H, magenta-colored neurons) 
(supplemental Movie 3, magenta-colored neurons, available at www.jneurosci.org as supplemental material) (64 neurons in five brains). On some occasions, multiple long processes elongated to some extent. However, eventually only one exceeded a length of $200 \mu \mathrm{m}$ (Fig. 2F) (supplemental Movie 4, available at www.jneurosci.org as supplemental material).

Axon-like processes appeared to preferentially extend radially (Fig. 2G,H, magenta-colored cells), with the majority toward the ventricle and a minority toward the pial surface (supplemental Movie 3, available at www.jneurosci.org as supplemental material). We analyzed the direction of the extension of the axon-like processes. The coronal plane was divided into four quadrants, and the direction was analyzed (Materials and Methods and supplemental Fig. S1, available at www.jneurosci.org as supplemental material). We found that a predominant population of labeled cells (59.9 \pm $12.8 \%)$ extended axon-like processes radially away from the pial surface ( $n=41$ of 64 cells, 5 brains), whereas a minority (21.1 \pm $9.3 \%$ ) extended toward the pial surface ( $n=14$ of 64 cells, 5 brains).

Some sea urchin-like cells did not extend axon-like processes during the observation period. The fact that the initiation of axon-like processes occurred at different time points among different cells, however, suggests that those cells would also elongate axon-like processes but at a later developmental stage.

We also observed rapidly migrating cells (supplemental Movie 3, arrowheads, available at www.jneurosci.org as supplemental material) among sea urchin-like cells, which are stationary. These migrating cells had leading processes, but the lengths of which never exceeded $200 \mu \mathrm{m}$, indicating that the rapidly extending process from sea urchin-like cells is distinct.

\section{In vivo preparations}

The peculiar features of the sea urchin-like cells and extension of axon-like processes raise the question as to whether these are in vitro artifacts or not. To test this possibility, we examined the morphology of labeled cells in fixed preparations not been subjected to culture. Observation of labeled cells in the CP at late embryonic and early postnatal days revealed cells with a remarkably similar morphology to the observed sea urchin-like cells (Fig. $3 A, B$ ), demonstrating that our results are not artifacts.

If the elongation of axon-like processes observed in the timelapse imaging occurs in vivo, we should be able to observe an increase in the number of cells with axons in fixed brains. As expected, we found that a majority of labeled cells observed in preparations fixed at $\mathrm{P} 1.5-\mathrm{P} 2.5$ had one process significantly longer than the rest (Fig. $3 C, D$, arrowheads). Moreover, a growthcone-like protrusion was observed at the leading edge of such long processes (Fig. 3C,D, circle). Figure 3, E and F, shows developmental changes in the length of the longest process and the proportion of labeled neurons that had a process $>200 \mu \mathrm{m}$ at each developmental stage. It should also be noted that both the length and proportion suddenly began to increase at P0.5, consistent with in vitro observations that found the initiation of the axonal growth takes place around this same stage.

These changes in the morphology of individual neurons occurred concomitant with changes in their orientation, from tangential to radial, and changes in the zonal distribution of labeled cells, from the MZ to the CP (supplemental Fig. S2, available at www.jneurosci.org as supplemental material), supporting the notion that extension of the axon-like process is related to the maturation of interneurons.

The labeled interneurons appeared to be a specific subset of interneurons, because they were primarily localized to specific layers, namely layers II/II and IV, when examined at P7. Interest-

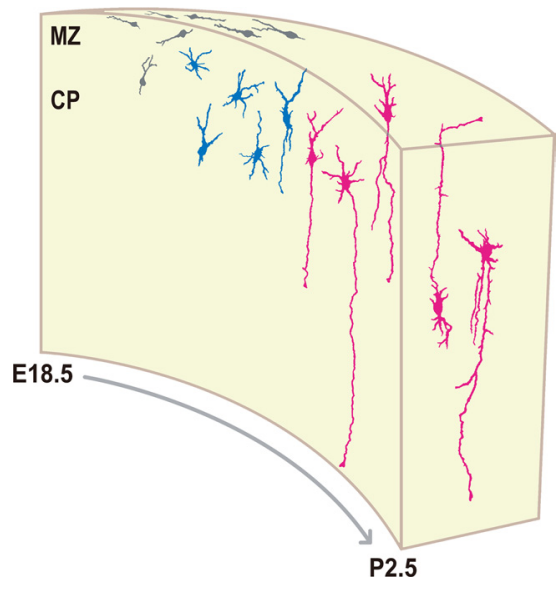

Figure 4. Schematic showing transitions of cortical interneuron morphology during perinatal development. Most cortical interneurons labeled by electroporation tangentially migrated in the MZ atE18.5. At this stage, they showed morphologies typical for migrating neurons extending a leading and a trailing process (gray). As development proceeds, they become situated in the $C P$, in which many of them alternately extend and retract of short processes (blue). These cells had a low motility of somata. Some of these cells then began to extend a long axon-like process (magenta) primarily toward the ventricle.

ingly, some of these cells looked like fast-spiking basket or double bouquet cells extending a vertically oriented axon (supplemental Fig. S3, available at www.jneurosci.org as supplemental material) (Uematsu et al., 2008). Immunostaining for PV, SST, or CR in P21 mice demonstrated that $\sim 60 \%$ of double-labeled cells expressed PV and 40\% expressed either SST or CR (data not shown).

\section{Discussion}

We have demonstrated that, during early postnatal development, cortical interneurons transiently exhibit sea urchin-like morphology, alternating between extended and retracted short processes, one of which suddenly elongated rapidly to transform into an axon-like morphology.

\section{Sea urchin-like cells}

We have demonstrated the occurrence of sea urchin-like interneurons in newborn mice cortex. Unlike migrating neurons observed at earlier stages (Nadarajah et al., 2002; Polleux et al., 2002; Tanaka et al., 2003, 2006, 2009), sea urchin-like cells alternately extend and retract many short processes without showing notable migration. It is unlikely that the sea urchin-like cell is an in vitro artifact because cells with similar morphologies were observed in fixed brains not subjected to culture (Fig. 3). The occurrence of neurons with similar morphologies in Golgi-impregnated preparations from kittens (Meyer and Ferres-Torres, 1984) supports this interpretation. Sea urchin-like cells remind us of multipolar cells reported in the embryonic cortex (Tabata and Nakajima, 2003) and cerebellar granule cells that settle in the inner granular layer (Kawaji et al., 2004). However, the former is distinct from sea urchin-like cells in that they are still in the course of migration. Moreover, the extension of axons from these cells has not been demonstrated. The latter also appears to be different in that they have a long axon-like leading process reaching the external granular layer, yet both have common features with our sea urchin-like cells and these two as all can completely or transiently cease migration. Therefore, one can surmise that neurons that stop migration, either transiently or permanently, 
exhibit nonpolarized morphologies and alternate between extending and retracting processes.

GABAergic interneurons are essentially bipolar in shape with a branched or unbranched leading process when they migrate in the subventricular zone and the MZ (Nadarajah et al., 2002; Polleux et al., 2002; Tanaka et al., 2003, 2006, 2009; Martini et al., 2009). These same neurons are likely to transform into sea urchin-like cells because the proportion of interneurons with a bipolar shape decrease during the perinatal stage in experiments in which interneurons were labeled at E12.5 (E.Y. and F.M., unpublished observation). Indeed, we occasionally observed the transformation of migrating GABAergic interneurons with a simple morphology into sea urchin-like cells (supplemental Movie 5, available at www.jneurosci.org as supplemental material).

\section{Initiation of axons}

After taking the sea urchin-like shape, cortical interneurons initiate thin, long processes. It is highly likely that these processes are prospective axons because (1) they continue to elongate while other processes repeat retractions and extensions, (2) they are headed by highly motile, growth-cone-like swellings during elongation, (3) they are significantly longer than other processes, (4) they do not taper off at their ends, and (5) they are longer than the leading processes of rapidly migrating neurons observed in the same preparation. In addition, the orientations of the prospective axon processes are similar to those of cortical interneuron subsets in the mature cortex such as fast-spiking basket, double bouquet, calretinin horizontal/descending arbor, and somatostatin cells (Uematsu et al., 2008).

Analysis of fixed samples further supports our interpretation. In fixed preparations, we observed labeled neurons with morphologies similar to those described above, and the length of the longest process increased during early postnatal development. Some of such a processes expressed an axonal marker (supplemental Fig. S4, available at www.jneurosci.org as supplemental material). Furthermore, our preliminary experiments in which the morphologies of labeled cells were analyzed at a later stage, P7, we found neurons with vertically oriented axons reminiscent of double bouquet (supplemental Fig. S3, available at www. jneurosci.org as supplemental material).

Together, the initiation of axons from interneurons likely occurs after cortical interneurons become sea urchin-like cells. However, we cannot unequivocally rule out the possibility that the long processes we observed eventually retract and that new processes emerge during a later stage.

In fixed samples, we observed a minor proportion of neurons whose axons extended toward the pial surface, some of which even reached marginal zone. Therefore, it is possible that, for these cells, the trailing process turns into the axon.

\section{Direction and orientation of axonal growth}

The reason the axons preferentially elongate either toward or away from the pial surface remains unknown. One possibility is environmental attractive cues. In Caenorhabditis elegans, a diffusible axon guidance molecule, UNC-6, induces ventrally directed polarity in neurons (Adler et al., 2006). Likewise, a secreted molecule, semaphorin $3 \mathrm{~A}$, regulates the axon-dendritic polarity in cortical pyramidal neurons in vitro (Polleux et al., 1998, 2000). It is possible that a similar mechanism determines the direction of axonal extensions seen in our study. However, we cannot preclude the possibility that interneurons have predetermined but morphologically undetectable polarity before initiation of axons.
Axonal growth from interneurons most often occurred along the radial axis in a very strict manner. Although the reason for this stereotyped orientation of growth remains unknown, it is possible that the axons use some physical substrate for their growth. Potential candidates include radial glial fibers and apical dendrites from excitatory neurons, because these processes strictly extend along the radial axis.

Our results are consistent with those from classic experiments done by Dotti and colleagues (1988) in which neurons exhibited a nonpolarized shape before the initiation of the axon. Given that many processes extend and retract before the axonogenesis (Fig. $2 F$ ) (supplemental Movie 4, available at www.jneurosci.org as supplemental material) and multiple processes extend on either the basal or apical side and that we found multiple axon-like processes elongated and retracted alternately until one unexpectedly became an axon-like process, it is likely that axonal fate of cortical interneurons is determined stochastically (Bradke and Dotti, 2000).

In this study, we show that, during perinatal development, cortical interneurons initiate an axon from a morphologically nonpolarized shape concurrently with changes in zonal distribution from the MZ to the CP (Fig. 4). This mode of axon initiation may be a widely occurring phenomenon but was first illustrated here using long-term live imaging of a cortical explant.

\section{References}

Adler CE, Fetter RD, Bargmann CI (2006) UNC-6/Netrin induces neuronal asymmetry and defines the site of axon formation. Nat Neurosci 9:511-518.

Anderson SA, Eisenstat DD, Shi L, Rubenstein JL (1997) Interneuron migration from basal forebrain to neocortex: dependence on Dlx genes. Science 278:474-476.

Arimura N, Kaibuchi K (2007) Abstract Neuronal polarity: from extracellular signals to intracellular mechanisms. Nat Rev Neurosci 8:194-205.

Bortone D, Polleux F (2009) KCC2 expression promotes the termination of cortical interneuron migration in a voltage-sensitive calcium-dependent manner. Neuron 62:53-71.

Bradke F, Dotti CG (2000) Establishment of neuronal polarity: lessons from cultured hippocampal neurons. Curr Opin Neurobiol 10:574-581.

Dotti CG, Sullivan CA, Banker GA (1988) The establishment of polarity by hippocampal neurons in culture. J Neurosci 8:1454-1468.

Goslin K, Banker G (1989) Experimental observations on the development of polarity by hippocampal neurons in culture. J Cell Biol 108:1507-1516.

Hinds JW, Hinds PL (1974) Early ganglion cell differentiation in the mouse retina: an electron microscopic analysis utilizing serial sections. Dev Biol 37:381-416.

Hinds JW, Hinds PL (1978) Early development of amacrine cells in the mouse retina: an electron microscopic, serial section analysis. J Comp Neurol 179:277-300.

Kawaji K, Umeshima H, Eiraku M, Hirano T, Kengaku M (2004) Dual phases of migration of cerebellar granule cells guided by axonal and dendritic leading processes. Mol Cell Neurosci 25:228-240.

Lambert de Rouvroit C, Goffinet AM (2001) Neuronal migration. Mech Dev 105:47-56.

Martínez S, Puelles L, Alvarado-Mallart RM (1992) Tangential neuronal migration in the avian tectum: cell type identification and mapping of regional differences with quail/chick homotopic transplants. Brain Res Dev Brain Res 66:153-163.

Martini FJ, Valiente M, López Bendito G, Szabó G, Moya F, Valdeolmillos M, Marín O (2009) Biased selection of leading process branches mediates chemotaxis during tangential neuronal migration. Development 136: 41-50.

Meyer G, Ferres-Torres R (1984) Postnatal maturation of nonpyramidal neurons in the visual cortex of the cat. J Comp Neurol 228:226-244.

Nadarajah B, Alifragis P, Wong RO, Parnavelas JG (2002) Ventricledirected migration in the developing cerebral cortex. Nat Neurosci 5:218-224.

Polleux F, Giger RJ, Ginty DD, Kolodkin AL, Ghosh A (1998) Patterning of 
cortical efferent projections by semaphorin-neuropilin interactions. Science 282:1904-1906.

Polleux F, Morrow T, Ghosh A (2000) Semaphorin 3A is a chemoattractant for cortical apical dendrites. Nature 404:567-573.

Polleux F, Whitford KL, Dijkhuizen PA, Vitalis T, Ghosh A (2002) Control of cortical interneuron migration by neurotrophins and PI3-kinase signaling. Development 129:3147-3160.

Stensaas LJ (1967a) The development of hippocampal and dorsolateral pallial regions of the cerebral hemisphere in fetal rabbits. I. Fifteen millimeter stage, spongioblast morphology. J Comp Neurol 129:59-69.

Stensaas LJ (1967b) The development of hippocampal and dorsolateral pallial regions of the cerebral hemisphere in fetal rabbits. II. Twenty millimeter stage, neuroblast morphology. J Comp Neurol 129:71-83.

Tabata H, Nakajima K (2003) Multipolar migration: the third mode of radial neuronal migration in the developing cerebral cortex. J Neurosci 23:9996-10001.

Tamamaki N, Fujimori KE, Takauji R (1997) Origin and route of tangentially migrating neurons in the developing neocortical intermediate zone. J Neurosci 17:8313-8323.

Tamamaki N, Yanagawa Y, Tomioka R, Miyazaki J, Obata K, Kaneko T (2003) Green fluorescent protein expression and colocalization with calretinin, parvalbumin, and somatostatin in the GAD67-GFP knock-in mouse. J Comp Neurol 467:60-79.
Tanaka DH, Maekawa K, Yanagawa Y, Obata K, Murakami F (2006) Multidirectional and multizonal tangential migration of GABAergic interneurons in the developing cerebral cortex. Development 133:2167-2176.

Tanaka DH, Yanagida M, Zhu Y, Mikami S, Nagasawa T, Miyazaki J, Yanagawa Y, Obata K, Murakami F (2009) Random walk behavior of migrating cortical interneurons in the marginal zone: time-lapse analysis in flat-mount cortex. J Neurosci 29:1300-1311.

Tanaka D, Nakaya Y, Yanagawa Y, Obata K, Murakami F (2003) Multimodal tangential migration of neocortical GABAergic neurons independent of GPI-anchored proteins. Development 130:5803-5813.

Tsai LH, Gleeson JG (2005) Nucleokinesis in neuronal migration. Neuron 46:383-388.

Uematsu M, Hirai Y, Karube F, Ebihara S, Kato M, Abe K, Obata K, Yoshida S, Hirabayashi M, Yanagawa Y, Kawaguchi Y (2008) Quantitative chemical composition of cortical GABAergic neurons revealed in transgenic venus-expressing rats. Cereb Cortex 18:315-330.

Wentworth LE, Hinds JW (1978) Early motoneuron formation in the cervical spinal cord of the mouse: an electron microscopic, serial section analysis. J Comp Neurol 177:611-634.

Wilcock AC, Swedlow JR, Storey KG (2007) Mitotic spindle orientation distinguishes stem cell and terminal modes of neuron production in the early spinal cord. Development 134:1943-1954. 